- Chain/Tree Architectures: Chain/tree architectures have units that are connected together in a string or tree topology. This chain or tree can fold up to become space filling, but the underlying architecture is serial. Through articulation, chain architectures can potentially reach any point or orientation in space, and are therefore more versatile but computationally more difficult to represent and analyze and more difficult to control.

- Mobile Architectures: Mobile architectures have units that use the environment to maneuver around and can either hook up to form complex chains or lattices or form a number of smaller robots that execute coordinated movements and together form a larger "virtual" network.

Control of all three types of modular systems can be centralized or distributed among the modules, and can be executed in series or in parallel. Though most systems implemented today are composed of rigid components, compliant mechanisms and deformable units have also been explored.

Modular robotic systems can also be classified according to the way in which units are reconfigured (moved) into place.
- Deterministic Reconfiguration: This type of reconfiguration relies on units moving or being directly manipulated into their target location during reconfiguration. The exact location of each unit is known at all times or can be discovered and calculated at run time, and reconfiguration times can be guaranteed. Feedback control is often necessary to assure precise manipulation, for example, in the chain and mobile architecture. Macroscale systems are usually deterministic.

- Stochastic Reconfiguration: This type of reconfiguration relies on units moving around using statistical processes (like Brownian motion). The exact location of each unit is only known when it is connected to the main structure, but it may take unknown paths to move between locations. Reconfiguration times can be guaranteed only statistically. Stochastic architectures are more favorable at microscales. The environment, whether natural or manmade, provides much of the energy for transporting modules around in this type of system.

Other modular robotic systems exist that are not selfreconfigurable, and thus do not formally belong to this

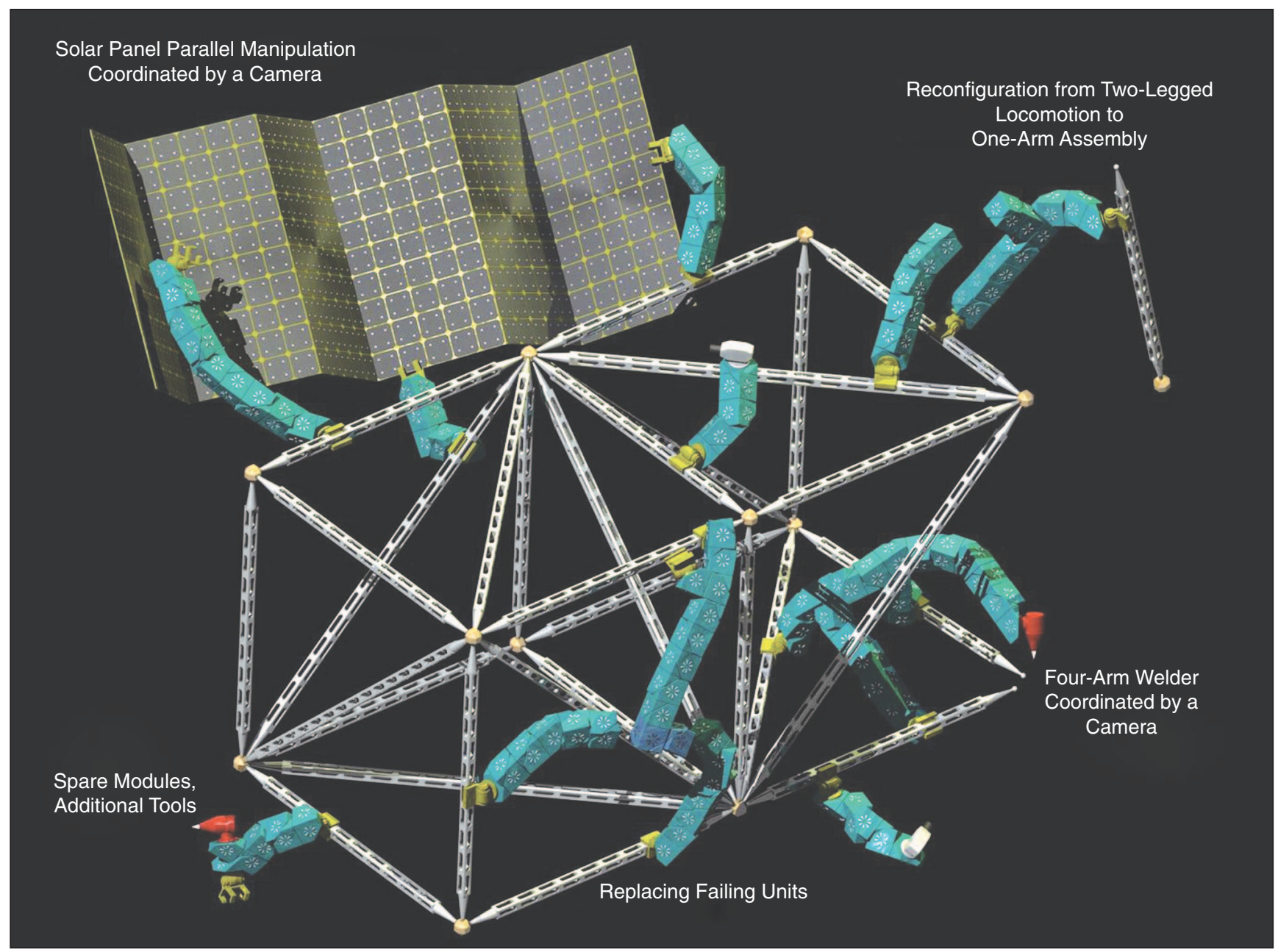

Figure 1. Artist rendition of a space application of modular robotics, showing a truss-building colony of chain/tree robots composed of cubical modules, configured in various morphologies for a variety of tasks including assembly, cooperative manipulation, and self-repair (from [1]). 
family of robots, though they may share similar design and control challenges. For example, self-assembling systems may be composed of multiple modules but cannot dynamically control or reconfigure their target shape. Tensegrity robots may be composed of multiple interchangeable modules, but cannot self-reconfigure. Swarm robots are composed of multiple units, but do not typically connect to form more complex physical structures. Even industrial robots with tool changers can be considered modular, but the degree to which they self-reconfigure is very limited in comparison with the kinds of systems reviewed in this article.

\section{Motivation and Inspiration}

There are three key motivations for designing modular selfreconfigurable robotic systems.

- Versatility: Self-reconfigurable robotic systems are potentially more adaptive than conventional systems. The ability to reconfigure allows a robot or a group of robots to disassemble and reassemble machines to form new morphologies that are better suited for new tasks, such as changing from a legged robot to a snake robot and then to a rolling robot.

- Robustness: Since robot parts are interchangeable (within a robot and between different robots), machines can also replace faulty parts autonomously, leading to self-repair.

- Low Cost: Self-reconfigurable robotic systems can potentially lower overall robot cost by making many copies of one (or relatively few) type of modules so economies of scale and mass production come into play. Also, a range of complex machines can be made from one set of modules, saving costs through reuse and generality of the system.

These three advantages have not yet been fully realized. The added degrees of freedom make modular robots more versatile in their potential capabilities, but also incur a performance tradeoff and increased mechanical and computational complexities. A modular robot is likely to be inferior in performance to any single custom robot tailored for a specific task. Consequently, the advantage of modular robotics is only apparent when considering multiple tasks that would normally require a set of different fixed-morphology robots, or when the nature of tasks cannot by fully determined before the robots are deployed.

\section{Application Areas}

Given these advantages, where would a modular selfreconfigurable system be used? While the system has the promise of being capable of doing a wide variety of tasks, finding the "killer application" has been somewhat elusive. Here are several examples.

\section{Space Exploration}

Long-term space missions (Figure 1) require a self-sustaining robotic ecology that can handle unforeseen situations and may require self-repair. Self-reconfigurable systems are

\section{Modular self-reconfigurable robotic systems can be generally classified into several architectural groups by the geometric arrangement of their units.}

better able to handle tasks that are not known a priori, especially compared to fixed-configuration systems. In addition, space missions are highly volume and mass constrained. Sending a robot system that can reconfigure to achieve many tasks saves shipping mass and volume as compared to sending many robots that each can accomplish one task.

\section{Bucket of Stuff}

A third long-term vision for these systems has been called "bucket of stuff." In this vision, consumers of the future have a container of self-reconfigurable modules. When the need arises, the consumer calls forth the robots to achieve a task such as "clean the gutters" or "change the oil in the car," and the robot assumes the shape needed and does the task.

One source of inspiration for the development of these systems comes from envisioned applications. A second source of inspiration originates in biological systems that self-construct out of a relatively small repertoire of lowerlevel building blocks (cells or amino acids, depending on the scale of interest). This architecture underlies the ability of biological systems to physically adapt, grow, heal, and even self-replicate-capabilities that would be desirable in many engineered systems.

\section{History and State of the Art}

The roots of the concept of modular self-reconfigurable robots can be traced back to the "quick change" end effecter and automatic tool changers in computer numerical controlled machining centers in the 1970s. Here, special modules, each with a common connection mechanism, could be automatically swapped out on the end of a robotic arm. Taking the basic concept of the common connection mechanism and applying it to the whole robot was introduced by Toshio Fukuda with the CEBOT (short for cellular robot) [2] in the late 1980s.

The early 1990s saw further development from Greg Chirikjian, Mark Yim, and Satoshi Murata. Chirikjian and Murata developed lattice reconfiguration systems, while Yim developed a chain-based system. These researchers started with a mechanical engineering emphasis, designing and building modules and then developing code to program them. The work of Daniela Rus and Wei-Min Shen developed hardware, but had a greater impact on the programming aspects. They started a trend towards provable or verifiable 
distributed algorithms for the control of large numbers of modules, as well as for dynamically discovering topological changes and automatically shifting behaviors according to new topologies.

One of the more interesting hardware platforms recently developed has been the modular transformer (MTRAN) series by Satoshi Murata et al. [3]. This system is a hybrid chain and lattice system. It has the best of both systems: the good task performance of a chain system mixed with the good reconfiguration performance of a lattice system.

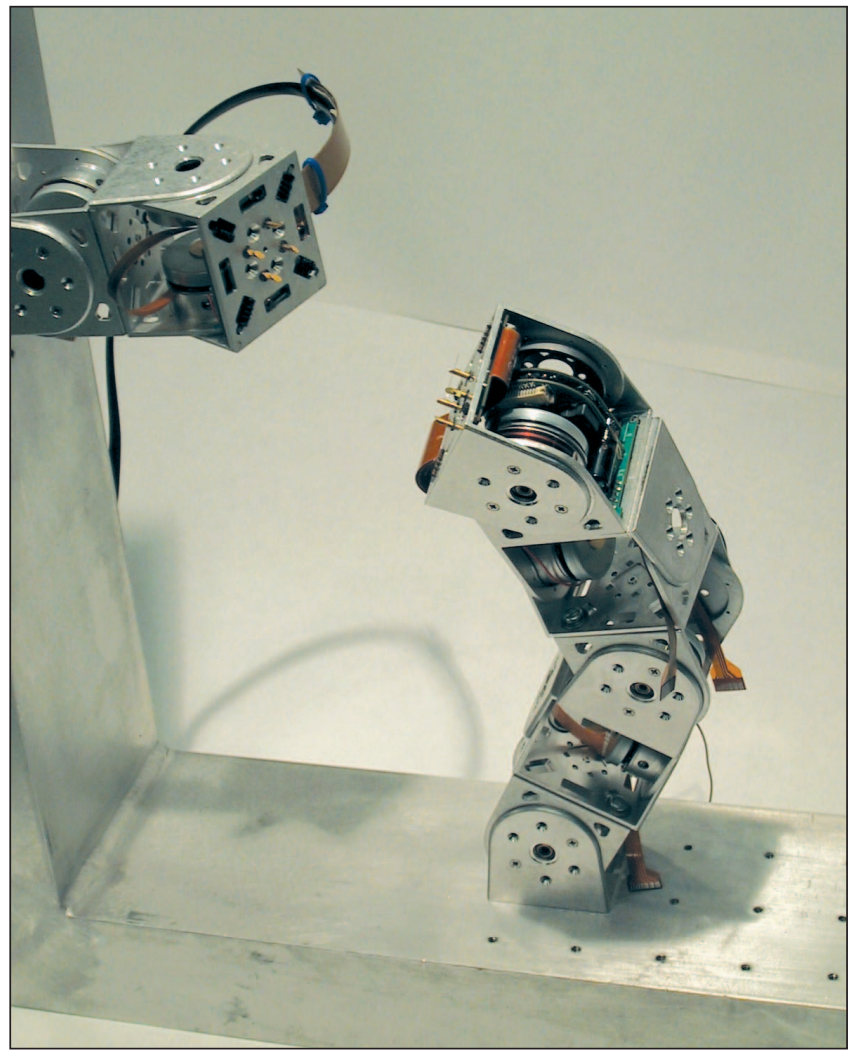

Figure 2. PolyBot G3 from PARC.

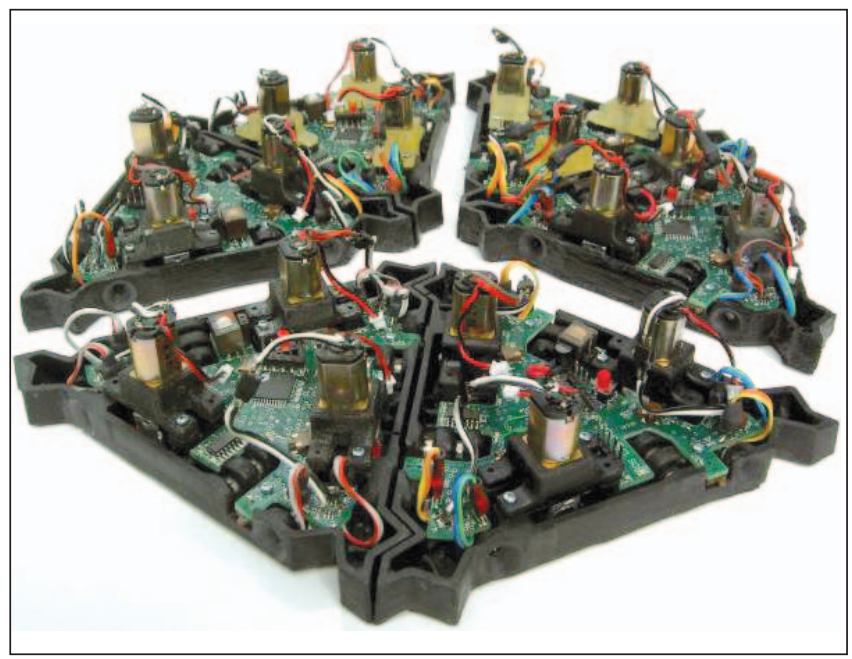

Figure 3. Self-organizing programmable parts from the University of Washington.
More recently, new efforts in stochastic self-assembly have been pursued by Hod Lipson and Eric Klavins. Thousands of modules have been simulated, with some (less than ten) hardware module demonstrations as well. These works build on demonstrations of Penrose dating back to the 1950s, and more recently the work of chemist $\mathrm{G}$. Whitesides.

A large effort at Carnegie Mellon University (in collaboration with Intel Research Pittsburgh) headed by Seth Goldstein and Todd Mowry has started looking at issues in developing millions of modules [4], focusing on simplifying hardware and addressing scalability issues. So far, they use large numbers in simulation with a few hardware module prototypes.

Many tasks have been shown to be achievable, especially with chain reconfiguration modules. This demonstrates the versatility of these systems. However, the other two advantages - robustness and low cost-have not been demonstrated. In general, the prototype systems developed in the labs have been fragile and expensive, as would be expected during any initial development.

There is a growing number of research groups actively involved in modular robotics research, as can been seen in the survey paper [5], a survey chapter in [6], and two special issues in robotics journals [7] and [8]. A number of algorithmic advances have complemented hardware development. See, for example, [6]-[10] and [12]-[14].

\section{Example Self-Reconfigurable Systems}

\section{PolyBot G3 (2002)}

PolyBot, seen in Figure 2, was created at Palo Alto Research Center (PARC), formerly known as Xerox PARC, by Yim et al. [9]. It is a chain self-reconfiguration system. Each module is roughly cubic shaped, about $50 \mathrm{~mm}$ on a side, and has one rotational degree of freedom (DOF). It is part of the PolyBot modular robot family that has demonstrated many modes of locomotion including: walking: biped, 14 legged, slinky-like; snake-like: concertina in a gopher hole, inchworm gaits, rectilinear undulation, and sidewinding gaits; rolling like a tread at up to $1.6 \mathrm{~m} / \mathrm{s}$; riding a tricycle; and climbing: stairs, poles, pipes, ramps, etc. The modules have brushless flat motors with harmonic drive transmission, force torque sensors, whisker touch sensors, and infrared proximity sensors. They use hermaphroditic connectors with shape memory alloy actuated latches.

\section{The Programmable Parts (2005)}

Figure 3 shows a testbed built by Klavins et al. at the University of Washington to explore what amount to programmable chemical reactions [10]. The programmable parts are stirred randomly on an air hockey table by randomly actuated air jets. When they collide and stick, they can communicate and decide whether to stay stuck or if and when to detach. Local interaction rules can be devised and optimized to guide the robots to make any desired global shape. The system, programmed by local rules, can be modeled using the chemical master equation and the analysis of its 
behavior follows standard ideas in nonequilibrium statistical dynamics. The resulting theory being developed is being applied to microscale self-assembly and even molecular selfassembly. The ultimate goal is to understand how to program stochastic self-assembly at all scales.

\section{Molecubes (2005)}

Figure 4 shows the Molecube system developed by Zykov et al. at Cornell University [1], built to physically demonstrate kinematic self-reproduction. Each module is a 0.65$\mathrm{kg}$ cube with 100-mm long edges and one rotational DOF. The axis of rotation is aligned with the cube's longest diagonal. Physical self-reproduction of a three- and a fourmodule robot was demonstrated, and the theoretical existence of arbitrarily sized self-replicating machines has been mathematically demonstrated. Other self-replicating morphologies and controllers have been shown to emerge spontaneously in a simulation of a "primordial soup" of thousands of 2-D Molecubes automata.

\section{SuperBot (2006)}

The SuperBot, seen in Figure 5, has been developed by Shen et al. at the University of Southern California as a deployable self-reconfigurable robot for real-world applications outside laboratories. Its modules have a hybrid chain and lattice architecture [11]. The modules have three DOF (pitch, yaw, and roll) and can connect to each other through one of the six identical dock connectors. They can communicate and share power through their dock connectors. For high-level communication and control, the modules use a real-time operating system and the hormone-inspired control developed for CONRO [12] as a distributed, scalable protocol that does not require the modules to have unique IDs. Movies for CONRO and SuperBot can be found at http://www.isi.edu/robots/.

\section{Miche (2006)}

The Miche system, shown in Figure 6, has been developed by Rus et al. at MIT. It is a modular lattice system capable of arbitrary shape formation. This system achieves self-assembly by disassembly and has demonstrated robust operation over hundreds of experiments. Each module is an autonomous robot cube capable of connecting to and communicating with its immediate neighbors. The connection mechanism is provided by switchable magnets. The modules use face-toface communication implemented with an infrared system to detect the presence of neighbors. When assembled into a structure, the modules form a system that can be virtually sculpted using a computer interface and a distributed process. The group of modules collectively decides who is and is not on the final shape using algorithms that minimize the information transmission and storage. Finally, the modules not in the structure let go and fall off under the control of an external force, in this case gravity. All the algorithms controlling these processes are distributed and are very efficient in their space and communication consumption.

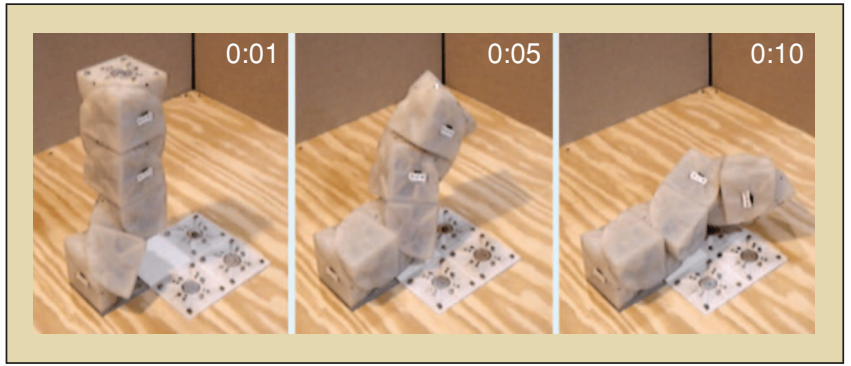

Figure 4. Molecube system from Cornell.

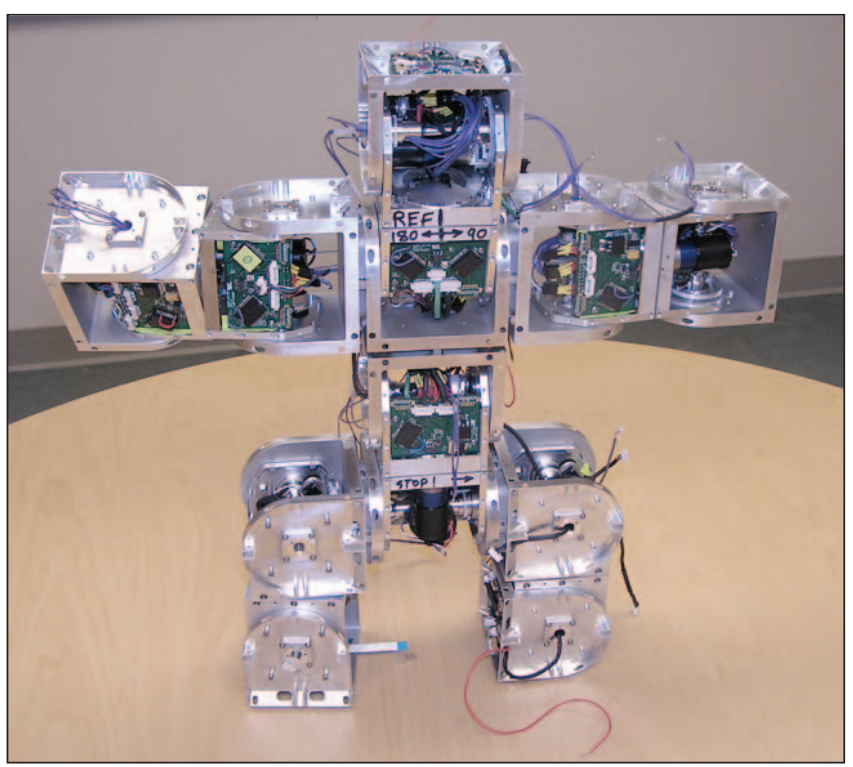

Figure 5. SuperBot from USC/ISI.

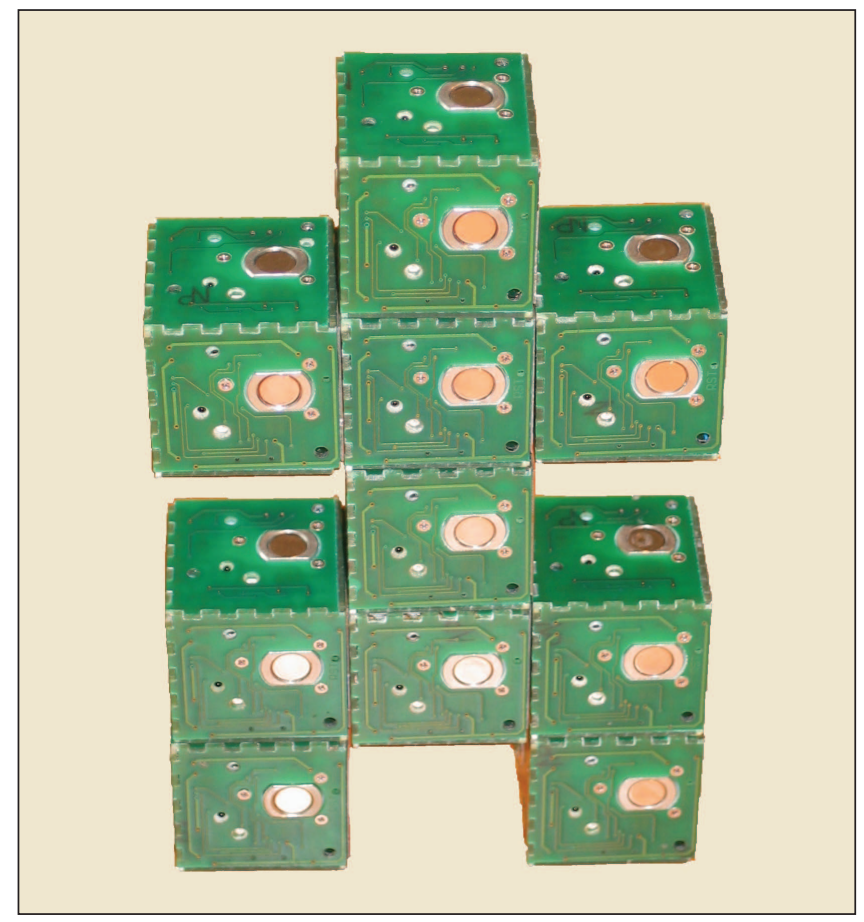

Figure 6. Miche from MIT. 


\section{Self-reconfigurable robots are also able to deliberately change their own shape by rearranging the connectivity of their parts.}

Table 1 lists many of the other instantiated modular robot systems. In addition to the name, class, and author, the table lists DOF. This describes the number of actuated DOFs for module motion (e.g., not latch DOFs) as well as whether the system motion is planar (2-D) or 3-D. The year is the estimated first public disclosure.

\section{Challenges for the Future}

\section{Challenges and Opportunities}

Since the early demonstrations of modular self-reconfigurable systems, size, robustness, and performance have been continuously improving. The extent to which the promise of selfreconfigurable robotic systems can be realized depends on the number of modules in the system. To date, only systems with up to about 50 units have been demonstrated, with this number stagnating for over almost a decade.
In parallel, planning and control algorithms have been progressing to handle millions of units. There are, however, several key steps that are necessary for these systems to realize their promise of adaptability, robustness, and low cost. These steps can be broken down into challenges in the hardware design, in planning and control algorithms, and in application.

\section{Hardware Design Challenges}

Performance of a self-reconfigurable robot is highly dependent on its mechanical and electronic control design. To date, a number of different designs have been developed and evaluated. Each design has primarily focused on some factors such as flexible form factor, utilizing many degrees of freedom, high torque-to-weight ratio, ease of docking/undocking, and power management. However, an optimal and general-purpose module design is yet to be proposed. The fundamental limiting factors that govern this problem include:

- limits on strength, precision, and field robustness (both mechanical and electrical) of bonding/docking inter

faces between modules

- limits on motor power, motion precision, and energetic efficiency of modules (i.e., specific power, specific torque)

- limits on dexterity of individual modules, which limits the flexibility of the robot as a whole.

Table 1. List of self-reconfigurable modular systems.

\begin{tabular}{|c|c|c|c|c|c|}
\hline System & Class & DOF & Author & Affiliation & Year \\
\hline CEBOT & mobile & various & Fukuda et al. & Nagoya & 1988 \\
\hline Polypod & chain & $23-D$ & Yim & Stanford & 1993 \\
\hline Metamorphic & lattice & $32-D$ & Chirikjian & JHU & 1993 \\
\hline Fracta & lattice & $32-D$ & Murata & MEL & 1994 \\
\hline Tetrobot & chain & $13-D$ & Hamlin et al. & RPI & 1996 \\
\hline 3D Fracta & lattice & $63-D$ & Murata et al. & MEL & 1998 \\
\hline Molecule & lattice & $43-D$ & Kotay \& Rus & Dartmouth & 1998 \\
\hline CONRO & chain & $23-D$ & Will \& Shen & USC/ISI & 1998 \\
\hline PolyBot & chain & $13-D$ & Yim et al. & PARC & 1998 \\
\hline TeleCube & lattice & $63-D$ & Suh et al. & PARC & 1998 \\
\hline Vertical & lattice & 2-D & Hosakawa et al. & Riken & 1998 \\
\hline Crystal & lattice & $42-D$ & Vona \& Rus & Dartmouth & 1999 \\
\hline I-Cube & lattice & $3-D$ & Unsal & $\mathrm{CMU}$ & 1999 \\
\hline Pneumatic & lattice & $2-D$ & Inoue et al. & TiTech & 2002 \\
\hline Uni Rover & mobile & $22-D$ & Hirose et al. & TiTech & 2002 \\
\hline MTRAN II & hybrid & $23-D$ & Murata et al. & AIST & 2002 \\
\hline Atron & lattice & $13-D$ & Stoy et al. & U.S Denmark & 2003 \\
\hline Swarm-bot & mobile & $32-D$ & Mondada et al. & EPFL & 2003 \\
\hline Stochastic 2D & stochastic & 0 2-D & White et al. & Cornell U. & 2004 \\
\hline Superbot & hybrid & $33-D$ & Shen et al. & USC/ISI & 2005 \\
\hline Stochastic 3D & stochastic & 0 3-D & White et al. & Cornell U. & 2005 \\
\hline Catom & lattice & $02-D$ & Goldstein et al. & CMU & 2005 \\
\hline Prog. parts & stochastic & $02-D$ & Klavins & U. Washington & 2005 \\
\hline Molecube & chain & $13-D$ & Zykov et al. & Cornell U. & 2005 \\
\hline YaMoR & chain & $12-D$ & ljspeert et al. & EPFL & 2005 \\
\hline Miche & lattice & $03-D$ & Rus et al. & MIT & 2006 \\
\hline
\end{tabular}




\section{Planning and Control Challenges}

Though algorithms have been developed for handling millions of units under specific ideal conditions, challenges to scalability remain both in low-level control and in high-level planning to overcome realistic constraints such as:

- algorithms for parallel motion for large-scale manipulation and locomotion with and without obstacles,

- algorithms for optimal (time, energy) reconfiguration planning with and without obstacles,

- algorithms for robustly handling a variety of failure modes, from misalignments and dead units (not responding, not releasing) to units that behave erratically,

- algorithms that determine the optimal configuration for a given task and environment, and

- efficient and scalable (asynchronous) communication among multiple units.

Mixed Software and Hardware Challenges

Self-reconfigurable systems arguably have more tightly coupled hardware and software than any other existing system. So, there are many issues for which solutions can
Over the last two decades, the field of modular robotics has advanced from proof-of-concept systems to elaborate physical implementations and simulations.

lie in either or both hardware and software classification. These issues include:

- The role of sensors in self-reconfigurable robotics: For many applications, the modules in a self-reconfigurable robot should be cognizant of their environment and their own state through a series of sensors. Due to the distributed nature of the network of modules, sensory information is available in a distributed form and, hence, this information must be fused for autonomous decisionmaking or communicated to a remote controller host.

\section{Table 2. Quantitative hardware achievements.}

\begin{tabular}{|c|c|c|c|c|c|}
\hline Accomplishment & Robot & Author & Affiliation & Quantity & Units \\
\hline $\begin{array}{l}\text { Most active modules in } \\
\text { connected system }\end{array}$ & PolyBot & Yim et al. & PARC & 56 & modules \\
\hline Smallest actuated module & Miniature & Yoshida et al. & AIST & $40 \times 40 \times 50$ & $\mathrm{~mm}$ \\
\hline Largest actuated module & Helium Catoms & Goldstein et al. & CMU & 8 & $\mathrm{~m}^{3}$ \\
\hline Strongest actuation & Polybot & Yim et al. & PARC & 5 & modules cantilever \\
\hline Fastest modular robot system & CKBot rolling & Sastra et al. & U. Penn & 26 & module lengths/s \\
\hline $\begin{array}{l}\text { Longest distance running, } \\
\text { one charge }\end{array}$ & SuperBot & Shen et al. & USC/ISI & 750 & $\mathrm{~m}$ \\
\hline $\begin{array}{l}\text { Mobile unconnected } \\
\text { modules docking }\end{array}$ & Swarm-bot & Mondada et al. & IRIDIA & 16 & $\begin{array}{l}\text { connecting } \\
\text { components }\end{array}$ \\
\hline Most robust self-reconfiguration & MTRAN II & Murata et al. & AIST/TiTech & 14 & $\begin{array}{l}\text { nonrepeating } \\
\text { attach/detach } \\
\text { steps }\end{array}$ \\
\hline
\end{tabular}

Table 3. Quantitative software achievements.

\begin{tabular}{|c|c|c|c|c|c|}
\hline Accomplishment & Software & Author & Affiliation & Quantity & Units \\
\hline Most generic algorithm & CA planning & Rus et al. & MIT & $\begin{array}{l}\text { Instantiated on three } \\
\text { types See [13] }\end{array}$ & Systems \\
\hline $\begin{array}{l}\text { Tightest bounds for } \\
\text { reconfiguration with } \\
\text { volume-traveling units }\end{array}$ & $\begin{array}{l}\text { PAC planning } \\
\text { for crystal }\end{array}$ & $\begin{array}{l}\text { Rus, Vona, } \\
\text { and Butler }\end{array}$ & Dartmouth & $\begin{array}{l}\text { O(1) for repositioning } \\
\text { one module }\end{array}$ & Time complexity \\
\hline $\begin{array}{l}\text { Tightest bounds for } \\
\text { surface-traveling } \\
\text { single-module } \\
\text { locomotion }\end{array}$ & $\mathrm{N} / \mathrm{A}$ & Chirikjian et al. & JHU & see [14] & see [14] \\
\hline $\begin{array}{l}\text { Most behaviors based } \\
\text { on topology }\end{array}$ & Hormone & Shen et al. & USC/ISI & 3 & Behaviors \\
\hline Largest simulated system & $\begin{array}{l}\text { Million module } \\
\text { march }\end{array}$ & Butler and Fitch & RIT and NICTA & 2.2 mil. & \# of modules \\
\hline
\end{tabular}


The added degrees of freedom

make modular robots more

versatile in their potential

capabilities, but also incur a

performance tradeoff and increased

mechanical and computational

complexities.

- Interaction with obstacles in real-world applications: In real-world applications, self-reconfigurable robots are required to perform locomotion, manipulation, and self-reconfiguration tasks in the presence of obstacles and in an uncontrolled environment.

\section{Quantitative Accomplishments}

So far, some of the challenges listed above have been met to some degree. Some of the more quantitative accomplishments are listed in Table 2 for hardware and Table 3 for software.

Note that the maximum number of hardware modules is lagging by several orders of magnitude behind algorithmic capabilities, suggesting that many of the barriers to physical scalability remain unaddressed. Both technical improvements, such as better bonding mechanisms, and conceptual progress, such as stochastic control, self-repair, and parallel manipulation, may ultimately play out in quantitative improvements, such as dramatically increasing the number of modules or making smaller ones.

\section{Application Challenges}

Besides the technical challenges, there are nontechnical challenges as well. Though the advantages of modular selfreconfigurable robotic systems have largely been established, it has been difficult to identify specific application domains where those benefits can be demonstrated. Many of the researchers developing this field have determined that finding an application that clearly drives the need for these systems is one of the major challenges.

\section{Grand Challenges}

The research described in this article represents an ultimate goal shared by the authors that modular robots may one day be used in vast numbers for practical applications where unsupervised, adaptive self-organization is crucial. Several key technical difficulties stand in the way, however. In this section, we describe several grand challenges that, if overcome, would enable a next generation of modular robots with vastly superior capabilities.

- Big systems: Most systems of modular robots have been small in number, especially compared to, for example, the number of components in a living cell (which many researchers view as the best example of a self-organizing modular system). The demonstration of a system with at least 1,000 individual units would suggest that modular robots have come of age. The physical demonstration of such a system will require rethinking key hardware issues, such as binding mechanisms, power distribution, dynamics, and vibrations. It will also require new distributed algorithms that account for noise, errors, failures, and changing connection topologies.

- Self-repairing systems: Besides reconfiguring itself into a new shape, a system comprised of modular robots would be able to recover from serious damage, such as that which might result from an external collision or internal failure. A demonstration of a selfhealing structure made up of many distributed, communicating parts would require rethinking algorithms for sensing and estimation of the global state, as well as truly robust hardware and algorithms for reconfiguration that work from any initial condition. A concrete example would be having a system blown up (randomly separated into many pieces) and then self-assemble, or recover from failure of a certain percentage of faulty units.

- Self-sustaining systems: Recently, NASA pushed a concept called Robosphere that looks at creating a self-sustaining robotic ecology, isolated for a long period of time, which needs to sustain operation and accomplish unforeseen tasks without any human presence. The current state of the art with modular robots is nowhere near this goal, and so a demonstration of a system actively running for, say, one year is crucial. New techniques in power management and energy harvesting would be required, as well as the ability to cope with the inevitable failures that would occur in such a long mission.

- Self-replication and self-extension: While simple robotic self-replication has been demonstrated using a few high-level modules, a significant challenge remains to demonstrate self-replication using many low-level modules, and ultimately from elementary components and even raw materials. Such a system could build active elements as well as passive structures, leading to a self-replicating and even self-improving system from environmental resources. The demonstration of a "seed" group of modular robots that can build copies of themselves from raw materials would require advancing beyond a level of complexity that Von Neumann identified as essentially the equivalent of the sound barrier for engineered systems.

- Reconciliation with thermodynamics: Modular robots are, in many ways, examples of the kinds of selforganizing systems studied by molecular biologists and nanotechnologists. However, there are key differences. Most existing systems overcome entropy through brute force and unreasonable amounts of 
energy. Molecular systems, on the other hand, employ random diffusive processes in fundamental ways. Furthermore, they are entirely robust to the intrinsic noise found at the nanoscale. If modular robots are to be miniaturized to micro- and/or nanoscale, or if the ideas discovered in this community are even to be tied to nanotechnology, the stochastic nature of nanoscale systems must be addressed. The demonstration of a system where stochastic fluctuations are the dominant factor would represent a fundamental advance: For example, pour a large collection (e.g., 1,000) of simple robots into a solution, mix them, and have them aggregate into a predetermined structure, independent of initial conditions.

A number of these issues were discussed in [15] (and the references therein) and will be addressed in greater detail in a forthcoming issue of IEEE Robotics \& Automation Magazine devoted to robotic self-replication and self-repair.

\section{Conclusions}

Modular self-reconfigurable systems have the promise of making significant technological advances to the field of robotics in general. Their promise of high versatility, high value, and high robustness may lead to a radical change in automation. Currently, a number of researchers have been addressing many of the challenges. While some progress has been made, it is clear that many challenges still exist. By illustrating several of the outstanding issues as grand challenges that have been collaboratively written by a large number of researchers in this field, this article has shown several of the key directions for the future of this growing field.

\section{Acknowledgments}

The authors wish to acknowledge the participation of all the attendees at the Robotics: Science and Systems workshop on self-reconfigurable systems, as well as the speakers and authors-Harris Chiu, Michael de Rosa, Haruhisa Kurokawa, Victor Zykov, Jason Campbell, Zack Butler, David Johan Christensen, Paulina Vashavskaya, Justin Werfel, Kiju Lee, Matt Moses, Michael Park, and Nicolas Brener.

\section{Keywords}

Modular, self-reconfigurable, self-reconfiguring, robot, grand challenges.

\section{References}

[1] V. Zykov, E. Mytilinaios, M. Desnoyer, and H. Lipson, "Evolved and designed modular robotics systems capable of self-reproduction," IEEE Trans. Robotics, to be published.

[2] T. Fukuda, S. Nakagawa, Y. Kawauchi, and M. Buss, "Self organizing robots based on cell structures-CEBOT," in Proc. IEEE/RSJ Int. Conf. Intelligent Robots and Systems (IROS), Nov. 1988, pp. 145-150.

[3] S. Murata, E. Yoshida, A. Kamimura, H. Kurokawa, K. Tomita, and S. Kokaji, "M-TRAN: Self-reconfigurable modular robotic system," IEEE/ASME Trans. Mech., vol. 7, no. 4, pp. 431-441. 2002.

[4] S.C. Goldstein, J.D. Campbell, and T.C. Mowry, "Programmable matter," IEEE Computer, vol. 28, no. 6, pp. 99-101, May 2005.

\section{Modular self-reconfigurable systems have the promise of high versatility, high value, and high robustness, making significant technological advances to the field of robotics that may lead to a radical change in automation.}

[5] R. Fitch, and D.L. Rus, "Self-reconfiguring robots in the USA," Japanese Robot. Soc. J., vol. 21, no. 8, pp. 4-10, Nov. 2003.

[6] E.H. Ostergaard, "Distributed control of the ATRON selfreconfigurable robot," Ph.D. thesis, Maersk McKinney Moller Institute for Production Technology, Univ. of Southern Denmark, Nov. 2004.

[7] D. Rus and G.S. Chirikjian, Eds., Autonom. Robots (Special Issue on Modular Robots), vol. 10, no. 1, 2001.

[8] W.-M. Shen and M. Yim, Eds., IEEE/ASME Trans. Mechatron. (Special Issue on Self-Reconfigurable Modular Robots), vol. 7, no 4, 2002.

[9] M. Yim, Y. Zhang, and D.G. Duff, "Modular Robots," IEEE Spectr., vol. 39, no. 2, pp. 30-34, Feb. 2002.

[10] E. Klavins, S. Burden, and N. Napp, "Optimal rules for programmed stochastic self-assembly," in Proc. Robotics: Science Systems '06, Aug. 2006.

[11] B. Salemi, M. Moll, and W.-M. Shen, "SUPERBOT: A deployable, multi-functional, and modular self-reconfigurable robotic system," in Proc. 2006 IEEE/RSJ Intl. Conf. Intelligent Robots Systems, Oct. 2006, pp. 3636-3641.

[12] W.-M. Shen, B. Salemi, and P. Will, "Hormone-inspired adaptive communication and distributed control for CONRO self-reconfigurable robots," IEEE Trans. Robotics Automat., vol. 18, no. 5, pp. 700-712, Oct. 2002.

[13] Z. Butler, K. Kotay, D. Rus, and K. Tomita, "Generic decentralized locomotion control for lattice-based self-reconfiguring robots," Int. J. Robotics Res., vol. 23, no. 9, pp. 919-938, 2004.

[14] G. Chirikjian, A. Pamecha, and I. Ebert-Uphoff, "Evaluating efficiency of self-reconfiguration in a class of modular robots," J. Robotic Systems, vol. 13, no. 5, pp. 317-338, May 1996.

[15] G.S. Chirikjian, Y. Zhou, and J. Suthakorn, "Self-replicating robots for lunar development," IEEE/ASME Trans. Mechatron., vol. 7, no. 4, pp. 462-472. Dec. 2002.

Mark Yim joined the Department of Mechanical Engineering and Applied Mechanics at U. Penn in the fall of 2004 as associate professor and Gabel Family Term Junior Professor. Prior to this, he was a principal scientist at the Palo Alto Research Center (formerly Xerox PARC), where he established a group developing modular self-reconfigurable robots. His other research interests include biologically inspired mechanisms, haptics for virtual reality, flying robots, and meso-scale MEMs devices. Honors include induction as a World Technology Network Fellow, IEEE Robotics and Automation Distinguished Lecturer, and induction to MIT's Technology Review TR100 in 1999. He has over 30 patents issued and over 50 publications.

Wei-Min Shen is the director of Polymorphic Robotics Laboratory at USC/Information Sciences Institute (ISI), associate director at the Center for Robotics and Embedded Systems, 
and a research associate professor in computer science at USC. He received his Ph.D. under Nobel Laureate Prof. Herbert A. Simon from Carnegie Mellon University in 1989. His research interests include self-reconfigurable and metamorphic systems, autonomous robots, machine learning, artificial intelligence, and life science. He has about 100 publications in these areas. $\mathrm{He}$ is the recipient the World Championship Award in 1997 Middle-Sized RoboCup Competition and a Phi Kappa Phi Faculty Recognition Award at USC in 2003. He is the inventor of hormone-inspired distributed and decentralized control for self-reconfigurable systems (U.S. Patent \#006636781).

Behnam Salemi is a computer research scientist at the University of Southern California, Information Sciences Institute. He received his B.S. degree in computer science from National University (Shahid Beheshti), Tehran, Iran, in 1991, and his M.S. and Ph.D. degrees in computer science and robotics from USC in 1997 and 2003, respectively. He is a member of the award-winning USC/ISI robotic team, the recipient of the gold medal in the international robotic soccer, and the recipient of the ISI Meritorious Award in 1997. His research activities have been reported by the media including Science, the L.A. Times, and CNN. His research interests include distributed multi-agent systems, distributed intelligent control, computer vision, distributed robotics, self-reconfigurable robots, and aerial robotics.

Daniela Rus is a professor in the EECS Department at MIT. She is the codirector of the CSAIL Center for Robotics. Previously, she was a professor in the Computer Science Department at Dartmouth College. She holds a Ph.D. degree in computer science form Cornell University. Her research interests include distributed robotics, mobile computing, and self-organization. She was the recipient of an NSF CAREER award and an Alfred P. Sloan Foundation Fellowship. She is a class of 2002 MacArthur Fellow.

Mark Moll is a research scientist at the Information Sciences Institute (ISI) in Marina del Rey, California. He received an M.S. degree in computer science from the University of Twente in The Netherlands in 1995. He received a Ph.D. degree in computer science from Carnegie Mellon University in 2002. His thesis work focused on shape reconstruction of unknown shapes using tactile data. His current research interests include self-reconfigurable robots, motion planning, shape reconstruction, shape representation, and computational biology.

Hod Lipson is an assistant professor at the departments of Mechanical and Aerospace Engineering and the faculty of
Computing and Information Science of Cornell University in Ithaca, New York. Prior to this appointment, he was a postdoctoral researcher at Brandeis University's Computer Science Department and a lecturer at MIT's Mechanical Engineering Department. He received his $\mathrm{Ph}$.D. from the Technion-Israel Institute of Technology in 1998. His research focuses on new methods for autonomous adaptation in behavior and morphology of robotic systems, with broader impacts to design automation and manufacturing technologies. His work uses primarily biologically inspired approaches, as they bring new ideas to engineering and new engineering insights into biology.

Eric Klavins is an assistant professor of electrical engineering at the University of Washington in Seattle. He received a B.S. degree in computer science in 1996 from San Francisco State University and M.S. and Ph.D. degrees in computer science and engineering in 1999 and 2001, respectively, from the University of Michigan in Ann Arbor, Michigan. From 2001-2003, he was a postdoctoral scholar in the Control and Dynamical Systems Department at the California Institute of Technology. As a graduate student, he was supported by a Charles DeVlieg Fellowship for manufacturing. In 2001, he received an NSF CAREER award titled "Programmable Robotic Self-Assembly." His research interests include cooperative control, robotics, distributed systems, concurrency, self-organizing systems, and nanotechnology.

Gregory S. Chirikjian received the B.S.E. degree in engineering mechanics, the M.S.E. degree in mechanical engineering, and the B.A. degree in mathematics, all from Johns Hopkins University. He received the Ph.D. degree from the California Institute of Technology, in 1992. Since the summer of 1992, he has been with the Department of Mechanical Engineering, Johns Hopkins University, where he is now professor and chair. He is a 1993 National Science Foundation Young Investigator, a 1994 Presidential Faculty Fellow, and a 1996 recipient of the ASME Pi Tau Sigma Gold Medal. His research interests include kinematic analysis, motion planning, design, and implementation of biologically inspired robots. In particular, "hyper-redundant," "metamorphic," "binary" manipulators, and, most recently, self-replicating robots. In recent years, he has also been applying methods from robotics to model conformational transitions in biological macromolecules.

Address for Correspondence: Mark Yim, 229A Towne Building, 220 S. 33rd Street, Philadelphia, PA 19104-6315 USA. Phone: +1 215898 5269. (e-mail: yim@grasp.upenn.edu). 